\title{
Construcción de un cuestionario de dilemas para indagar las concepciones sobre el aprendizaje a partir de la lectura en ingresantes universitarios
}

\section{Construction of a dilemmas questionnaire to investigate the conceptions of learning, based on the reading of first-year undergraduate students}

\author{
Yanina Elizabet Boatto ${ }^{1}$ \\ Consejo Nacional de Investigaciones Científicas y Tecnológicas y Ministerio de Ciencia \\ y Tecnología de Córdoba, Argentina \\ Universidad Nacional de Río Cuarto, Argentina \\ Gisela María Vélez ${ }^{2}$ \\ Adriana Irma Bono ${ }^{3}$ \\ Universidad Nacional de Río Cuarto, Argentina \\ (Rec: Noviembre 2010 - Acep: Marzo 2011)
}

\begin{abstract}
Resumen
En este trabajo presentamos el procedimiento de construcción de un cuestionario de dilemas que indaga las teorías implícitas de aprendizaje a partir de la lectura en estudiantes universitarios. Para su elaboración se consideraron dimensiones y categorías definidas desde desarrollos teóricos sobre teorías implícitas de aprendizaje y teorías científicas sobre la lectura. En tanto que desde la perspectiva empírica se atendió a las voces de los estudiantes; esto contribuyó a una formulación más ajustada tanto de los dilemas como de las diferentes opciones de resolución de los mismos, y orientó la selección de los escenarios educativos en los que éstos se enmarcan. La validez del instrumento se determinó mediante el proceso de control de expertos. Para establecer la confiabilidad se aplicó el coeficiente de Cronbach. Los índices de validez y confiabilidad obtenidos demuestran que el cuestionario está dentro de los valores requeridos, para ser administrado a los estudiantes.
\end{abstract}

Palabras clave: cuestionario de dilemas, concepciones de aprendizaje, lectura, ingresantes universitarios.

\begin{abstract}
In this work is presented the construction of a dilemmas questionnaire that explores the theories implicit of learning trought the reading in university students. In its preparation were considered dimensions and defined categories since developments theoretical above theories implicit of learning and scientific theories on the reading. In both from the perspective empirical were considered the voices of the students; this contributed to a more adjusted formulation both of the dilemmas as of the different options of resolution of the same, and steered the selection of educational scenarios in the which they fall. The validity of the instrument was determined through the process of experts control. To establish the reliability was applied the coefficient of Cronbach. The indexes of validity and reliability obtained show that the questionnaire is within the adequate values, for be administered to students.

Key words: dilemmas questionnaire, learning conceptions, reading, first-year undergraduate students.
\end{abstract}

1 Correspondencia a: Yanina Elizabet Boatto. Dirección Postal: Pasaje Potrerillo Nº73. Río Cuarto. Córdoba. República Argentina. C.P. 5800. Tel: 54-9-0358-4630889. E-mail: yaniboatto@hotmail.com; yboatto@hum.unrc.edu.ar.

2 Gisela María Vélez. Dirección Postal: Arturo M. Bas No 1123. Río Cuarto. Córdoba. República Argentina. C.P. 5800. Tel: 54-90358-46214978. E-mail: gvelez@hum.unrc.edu.ar.

3 Adriana Irma Bono. Dirección Postal: Llay Llay No 386. Banda Norte. Río Cuarto. Córdoba. República Argentina. C.P. 5800. Teléfono: 54-9-0358-4631175. E-mail: abono@hum.unrc.edu.ar. 


\section{Introducción}

Este trabajo forma parte de un proyecto de investigación referido a las concepciones sobre el aprendizaje a partir de la lectura en los ingresantes universitarios ${ }^{4}$. En los últimos años, nos hemos abocado a estudiar los cambios producidos en las concepciones sobre el aprendizaje -mediado por la lectura- que mantienen los estudiantes que ingresan a la universidad. En este sentido, nuestras investigaciones han ido mostrando la necesidad de construir instrumentos situados que permitan acceder al estudio de las concepciones de los alumnos, entendidas como teorías implícitas.

En atención a ello, nuestro propósito en este trabajo es presentar el proceso de construcción de un cuestionario de dilemas, que indaga las concepciones que mantienen los estudiantes universitarios sobre el aprendizaje a partir de la lectura de textos académicos.

Las concepciones sobre el aprendizaje a partir de la lectura como teorías implícitas

Los estudios sobre las concepciones de profesores y alumnos acerca del aprendizaje en diferentes dominios del conocimiento (físico, social, psicológico) se han constituido en un tópico relevante en las investigaciones psicológicas y educativas de la última década. La importancia de ahondar en estos trabajos reside en la hipótesis planteada desde los marcos teóricos constructivistas, acerca del fuerte papel mediador que ejercen las concepciones de los sujetos en el aprendizaje de diversos dominios (Rodrigo, Rodríguez \& Marrero, 1993; Pozo \& Scheuer, 1999; Pozo, Scheuer, Mateos \& Pérez Echeverría, 2006). El núcleo común de estas investigaciones encierra la idea de que las personas orientan su accionar en base a un sistema de creencias, conocimientos y valores que le confieren sentido, por lo tanto, desde la perspectiva educativa,

\footnotetext{
4 El proyecto integra el Programa Aprender en la Universidad. Motivación y representaciones de los estudiantes en el dominio del lenguaje escrito; aprobado y subsidiado por la Secretaría de Ciencia y Técnica de la Universidad Nacional de Río Cuarto. También, se inscribe en el Proyecto Enseñar y aprender en la universidad: concepciones y prácticas de lectura y escritura en contextos académicos, aprobado y subsidiado por Agencia Nacional de Promoción de Ciencia y Tecnología - FONCYT - PICT N 2391. En el marco de estos proyectos/programas, la primera autora desarrolla su tesis doctoral en carácter de Becaria de postgrado; beca financiada por el Consejo Nacional de Investigaciones Científicas y Tecnológicas, y el Ministerio de Ciencia y Tecnología de Córdoba. República Argentina.
}

constituyen una base para analizar la naturaleza y las condiciones de posibilidad de los procesos de cambio que promueve la enseñanza.

Gran parte de esas investigaciones consideran que las concepciones se organizan en teorías en gran medida implícitas. El carácter 'teórico' atribuido a las concepciones se refiere a un núcleo de supuestos subyacentes, de orden epistemológico, ontológico y conceptual, que les otorgarían cierto grado de coherencia; asimismo estos constructos serían de naturaleza procedimental, difíciles de controlar de manera consciente, lo que les da el carácter de 'implícitos' (Pozo et al., 2006; Atkinson \& Claxton, 2000; Pozo \& Scheuer 1999; Pozo \& Gómez Crespo, 1998; Rodrigo et al., 1993). En el marco de estos estudios, se podría entender que el aprendizaje a partir de la lectura está en parte condicionado por teorías implícitas sobre el aprendizaje en este dominio, puesto que éstas orientarían las prácticas desarrolladas por los lectores cuando leen para aprender.

Los estudios referidos a las teorías implícitas sobre el aprendizaje en distintos dominios del conocimiento, han descrito tres configuraciones denominadas teoría directa, teoría interpretativa y teoría constructiva. Estas configuraciones presentan características peculiares referidas al modo en que los sujetos entienden las condiciones, los procesos y los resultados del aprendizaje.

Sintéticamente, los estudios que describen estas tres teorías (Pozo et al., 2006; Pozo, 2009) nos muestran que los sujetos que mantienen una teoría directa, asumen una relación lineal entre las condiciones y los resultados del aprendizaje, es decir que bastaría la co-presencia del sujeto y objeto de conocimiento para que se produzca el aprendizaje, entendido como un estado de conocimiento (a nivel ontológico); además el resultado obtenido debe ajustarse al objeto (realismo epistemológico). En la teoría interpretativa, se reconoce la interacción entre sujeto y objeto de conocimiento por medio de la actividad mental del aprendiz, lo que da lugar a transformaciones del objeto (ontológicamente el aprendizaje se concibe como un proceso), pero los resultados siguen entendiéndose como ajustados a este último (epistemología realista). En otras palabras, desde esta concepción, aunque se considera que la realidad no puede aprehenderse de modo perfecto debido a la subjetividad del aprendiz, el conocimiento se concibe como único y verdadero. De otro modo, los sujetos que se ubican en la teoría constructiva entienden al aprendizaje como un proceso de construcción y reconstrucción de las representaciones del mundo y de sí mismos acerca 
del mundo, advirtiendo que los procesos que median entre condiciones y resultados promueven transformaciones del sujeto y del objeto de conocimiento, situación que se da en un contexto determinado que influye en dicho aprendizaje (ontológicamente el aprendizaje se concibe como un sistema); y si bien no se ignora al objeto, se considera que los resultados pueden asumir diferentes versiones (perspectivismo epistemológico).

Las relaciones entre sujeto y objeto de conocimiento planteadas en las teorías implícitas sobre el aprendizaje pueden vincularse conceptualmente con las relaciones entre lector, texto y contexto que se plantean en tres enfoques científicos de lectura: lectura como conjunto de habilidades, enfoque interactivo y enfoque transaccional (Dubois, 1987; Goodman, 1994; Rosenblatt 1994), y con las características que se atribuyen en cada uno de estos enfoques al proceso de comprensión del texto por parte del lector (Rinaudo, 1999; Braslavsky, 2005).

En vinculación con la teoría directa de aprendizaje, desde el enfoque que concibe a la lectura como conjunto de habilidades, se considera que el lector avanza en la lectura a partir de unidades del lenguaje, entendiendo al texto como un conjunto de signos. Así, va reconociendo lo que 'dice' el autor por medio de la decodificación y el resultado del aprendizaje se refleja en la reproducción literal de lo leído. De este modo, se producen actos mecánicos de lectura y la comprensión del texto sería una consecuencia "natural' de la decodificación (Dubois, 1987; Rinaudo, 1999; Braslavsky, 2005).

En relación con la teoría interpretativa de aprendizaje, desde el enfoque interactivo de lectura, se entiende que el lector no recepta unidades aisladas del texto sino que procesa estructuras o unidades mayorantes, significando las palabras y construyendo una representación mental del texto que se va modificando a medida que avanza en la lectura. El aprendizaje es el significado que 'se extrae' del texto, a partir de la apropiación del 'pensamiento' del autor; si bien se trata de intervenir para comprenderlo y desentrañar por lo tanto 'lo que quiere decir' el autor, se busca una única idea posible. De este modo, se desarrolla un proceso de comprensión de tipo literal, ya que el lector debe 'comprender' las ideas 'del autor' (Rinaudo, 1999; Braslavsky, 2005).

De modo diferente y vinculado a la teoría constructiva de aprendizaje, desde el enfoque transaccional de lectura, la comprensión es activa o inferencial, se da a partir del intercambio de ideas entre lector y autor, por medio del texto, en un contexto determinado. Las características personales, los propósitos del lector y el momento particular de lectura hacen que cada sujeto pueda construir un significado personal del texto en múltiples sentidos. La diferencia espacial, temporal y cultural entre lector y autor hace que la interpretación sea 'versátil', el texto se reconstruye en un nuevo contexto, aún cuando la interpretación requiera recuperar los contextos iniciales de producción. Asimismo, la construcción del significado es diferente según el tipo de texto, su variedad discursiva y su estructura (Rinaudo, 1999; Braslavsky, 2005).

Las teorías científicas sobre la lectura posibilitan un marco de indagación y contraste para el análisis de las concepciones acerca del aprendizaje mediado por el acto de leer. Como hemos expuesto, este aprendizaje estaría mediado por las concepciones implícitas de los sujetos y se hace necesario estudiarlas para promover su explicitación y reestructuración.

Las teorías implícitas difícilmente se exponen de modo declarativo, es por ello que sus características y supuestos se infieren a partir de las actuaciones y decisiones de los sujetos, de tal modo que para su estudio se requiere apelar a métodos indirectos. Diversas investigaciones dan cuenta de que el cuestionario de dilemas es uno de los instrumentos pertinentes para indagarlas, ya que el mismo se concentra en la activación de situaciones de aprendizaje específicas, correspondientes al dominio que se pretende estudiar (Macchiarola, 2008; Vilanova, García \& Señoriño, 2007; Pérez, Echeverría, Mateos, Scheuer \& Martín, 2006).

\section{El cuestionario de dilemas}

\section{a.1. Caracterización del instrumento}

El cuestionario de dilemas es un instrumento de indagación de carácter estructurado, que enfrenta al sujeto ante situaciones prácticas conflictivas referidas a un dominio de conocimiento y acerca de las cuales debe tomar decisiones (Macchiarola, 2004); se conforma básicamente por un conjunto de situaciones problemáticas y una serie de opciones de resolución para cada una de ellas.

Las situaciones problemáticas se sitúan en los contextos prácticos que enfrentan cotidianamente los sujetos. Para cada situación problemática se ofrece un grupo de opciones y el sujeto debe seleccionar aquella que considere más apropiada en términos de resolución del dilema. Las opciones se presentan mediante un discurso deliberativo y argumentativo que procura generar la adhesión del sujeto. Tanto 
las situaciones-problema como las opciones, se elaboran a partir de los constructos teóricos vinculados a un dominio de estudio en particular; atendiendo dentro de cada dominio a las dimensiones que lo constituyen (Macchiarola, 2008).

\section{a.2. Proceso de construcción}

El cuestionario de dilemas que construimos para investigar las concepciones de aprendizaje a partir de la lectura de textos académicos en ingresantes universitarios, se estructuró deductivamente a partir de tres dimensiones: 1) teoría directa, 2) teoría interpretativa y 3) teoría constructiva; y cuatro categorías para cada una de las dimensiones mencionadas: 1) concepto, 2) condiciones, 3) procesos y4) resultados de aprendizaje a partir de la lectura.

En la siguiente tabla se presenta condensadamente la conceptualización de las dimensiones y categorías que orientaron la elaboración del cuestionario de dilemas.

Tabla 1. Dimensiones y categorías del cuestionario de dilemas.

\begin{tabular}{|c|c|c|c|}
\hline $\begin{array}{ll}\text { Dimensiones } & \\
\text { Categorías }\end{array}$ & Teoría Directa & Teoría Interpretativa & Teoría Constructiva \\
\hline $\begin{array}{l}\text { Concepto de aprendizaje } \\
\text { Tipo de entidad que se le otor- } \\
\text { ga al conocimiento. ¿Qué es } \\
\text { aprender? }\end{array}$ & $\begin{array}{l}\text { Reproducción literal de lo leído: } \\
\text { aprender es expresar exactamen- } \\
\text { te lo que el texto dice. } \\
\text { Se prioriza un único resultado } \\
\text { correcto. }\end{array}$ & $\begin{array}{l}\text { Interpretación de la/s idea/s del } \\
\text { autor: aprender es expresar lo } \\
\text { que dice el texto, en función } \\
\text { de los procesos desarrollados } \\
\text { para ello. } \\
\text { Se prioriza un resultado correc- } \\
\text { to, pero éste se considera sujeto } \\
\text { al procedimiento de lectura, y } \\
\text { por lo tanto no se produce una } \\
\text { reproducción literal de lo leído. }\end{array}$ & $\begin{array}{l}\text { Construcción de un significado } \\
\text { personal del texto: aprender es } \\
\text { recrear el texto. } \\
\text { Se prioriza la atención a ca- } \\
\text { racterísticas del lector, texto y } \\
\text { contexto; considerando que la } \\
\text { transacción entre estos factores } \\
\text { posibilita distintas construccio- } \\
\text { nes de un mismo texto, por lo } \\
\text { que no habría un único resultado } \\
\text { posible sino un perspectivismo } \\
\text { en la interpretación. }\end{array}$ \\
\hline \begin{tabular}{|l|} 
Condiciones de aprendizaje \\
Factores del lector, texto y/o \\
contexto involucrados en el \\
aprendizaje. Así como el modo \\
en que el aprendiz considera que \\
operan en él. \\
¿Qué factores inciden en el \\
aprendizaje? ¿Cómo operan \\
esos factores?
\end{tabular} & $\begin{array}{l}\text { Presencia de factores no vincu- } \\
\text { lados, relativos al lector, texto } \\
\text { y/o ambiente físico. } \\
\text { Factores que operan como } \\
\text { determinantes. }\end{array}$ & $\begin{array}{l}\text { Factores referentes al lector y al } \\
\text { texto, vinculados. } \\
\text { Factores en los que intervienen } \\
\text { procesos mentales y afectivos. }\end{array}$ & $\begin{array}{l}\text { Interdependencia entre facto- } \\
\text { res relativos al lector, texto y } \\
\text { contexto. } \\
\text { Factores en los que intervienen } \\
\text { procesos mentales y afectivos } \\
\text { que provocan cambios en el } \\
\text { aprendizaje. }\end{array}$ \\
\hline $\begin{array}{l}\text { Proceso de aprendizaje } \\
\text { Actividad desarrollada para } \\
\text { poder aprender. } \\
\text { ¿Cómo se aprende? }\end{array}$ & $\begin{array}{l}\text { Actividad observable - acto } \\
\text { mecánico de lectura. } \\
\text { (Incorporación de la infor- } \\
\text { mación presente en el texto, } \\
\text { práctica y repetición a los fines } \\
\text { de la memorización). }\end{array}$ & $\begin{array}{l}\text { Actividad interpretativa-com- } \\
\text { prensión literal. } \\
\text { (Incorporación de información } \\
\text { presente en el texto leído, me- } \\
\text { diante procesos de selección, } \\
\text { organización, memorización, } \\
\text { control de lo memorizado, au- } \\
\text { tocorrección, etc.). }\end{array}$ & $\begin{array}{l}\text { Actividad constructiva - com- } \\
\text { prensión activa y crítica. } \\
\text { (Construcción y reconstrucción } \\
\text { del conocimiento, en base a la } \\
\text { reflexión, indagación, mirada } \\
\text { crítica, etc. frente al texto) }\end{array}$ \\
\hline $\begin{array}{l}\text { Resultado de aprendizaje } \\
\text { Producto del aprendizaje. } \\
\text { ¿Qué se aprende? }\end{array}$ & $\begin{array}{l}\text { Se aprende lo que el texto dice: } \\
\text { los contenidos literales expues- } \\
\text { tos en el texto. }\end{array}$ & $\begin{array}{l}\text { Se aprende lo que el texto repre- } \\
\text { senta: los conocimientos están } \\
\text { presentes en el texto y el lector } \\
\text { debe representárselos a sí mismo } \\
\text { para poder aprenderlos. }\end{array}$ & $\begin{array}{l}\text { Se aprende la representación que } \\
\text { el lector puede hacer del texto: el } \\
\text { lector reelabora los contenidos } \\
\text { para aprenderlos, construyendo } \\
\text { un texto paralelo. }\end{array}$ \\
\hline
\end{tabular}

Por otra parte, se avanzó en el análisis y justificación de decisiones referidas al control de la deseabilidad social, al lenguaje y a los escenarios requeridos para la construcción de los dilemas.

Con el propósito de disminuir el efecto de deseabilidad social en la elección de las opciones, se decidió proponer dilemas en los que no se involucrara personalmente a los sujetos, sino que se plantearon situaciones en las que los protagonistas son otros estudiantes (compañeros/pares), evitando así que los participantes deban juzgar sus propias actuaciones.

Asimismo, se atendió al lenguaje cotidiano de los ingresantes universitarios; para ello apelamos a estudios previos sobre concepciones de lectura, realizados en el mismo contexto, que proporcionaron fuentes 
'auténticas', provenientes de las respuestas de estudiantes de primer año de la universidad a preguntas formuladas en una entrevista semi-estructurada. Estos registros nos orientaron en el modo de expresión de los enunciados y en el vocabulario a ser utilizado en los dilemas. A partir de estas fuentes seleccionamos expresiones propias de los alumnos, ubicándolas en las distintas dimensiones y categorías del cuestionario. Se utilizó en este proceso el programa de análisis de datos cualitativos Atlas.ti. Posteriormente, la selección y categorización de las expresiones de los alumnos fueron controladas por expertos.

Otros aspectos considerados fueron los escenarios educativos en los cuales enmarcar los dilemas, procurando que éstos respondieran a los contextos propios que deben enfrentar los estudiantes en situaciones naturales de aprendizaje en la universidad (Pérez Echeverría, Pozo, Pecharromán, Cervi \& Martínez, 2006): leer para escribir con otros, leer para hacer una presentación oral individual y leer para ser evaluado.

Esto permitió construir una primera versión del cuestionario, conformado por doce situaciones dilemáticas y tres opciones de resolución para cada una de estas situaciones. Estas opciones representan a las dimensiones del cuestionario, las teorías implícitas de aprendizaje: teoría directa, teoría interpretativa y teoría constructiva. Para cada una de estas dimensiones, como hemos mencionado precedentemente, se consideraron cuatro categorías: concepto, condiciones, procesos y resultados de aprendizaje a partir de la lectura. Asimismo, las dimensiones y categorías se contextualizaron en los tres escenarios educativos señalados: leer para escribir con otros, leer para hacer una presentación oral individual y leer para ser evaluado.

La organización de las situaciones dilemáticas siguió la estructura que se presenta a continuación. La tabla 2 indica que TD corresponde a teoría directa, TI a teoría interpretativa y TC a teoría constructiva. En tanto que A- refiere al escenario 'leer para escribir con otros', B- a 'leer para hacer una presentación oral individual' y C- a 'leer para ser evaluado'. El ordenamiento numérico 1-12 da cuenta de las doce situaciones dilemáticas.

Tabla 2. Estructura de organización de las situaciones dilemáticas.

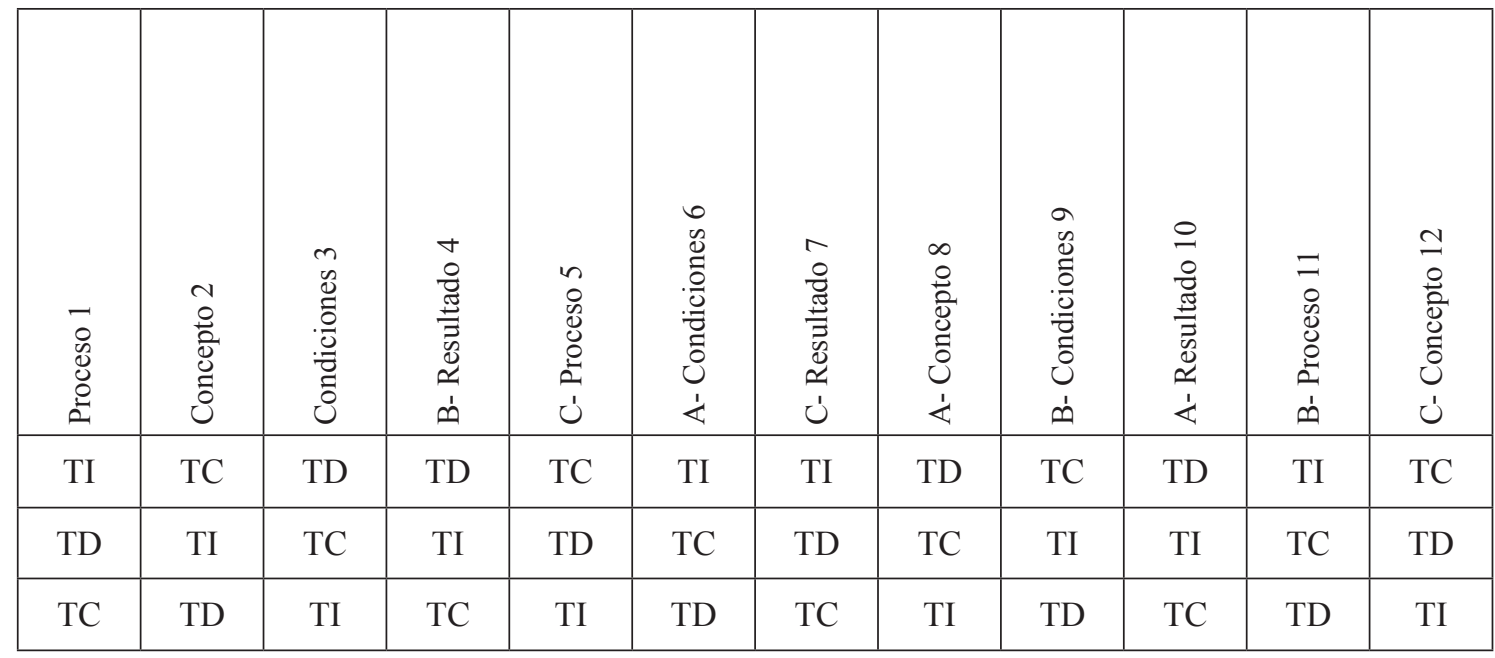

\section{a.2.1. Situaciones dilemáticas y opciones de resolución}

A continuación se muestran algunos ejemplos de las situaciones dilemáticas y sus correspondientes opciones de resolución, construidas para cada una de las categorías trabajadas, atendiendo también a los diferentes escenarios educativos.

- Para la categoría concepto de aprendizaje a partir de la lectura y el escenario leer para hacer una presentación oral individual:
En una materia de primer año universitario, al finalizar el año, el profesor le pide a sus alumnos que expongan oralmente las ideas propuestas por un autor, que fueron aprendidas a lo largo de la materia. Les da una hora para organizar la exposición. No todos los alumnos opinan lo mismo sobre cómo organizarla ¿Con quién estás más de acuerdo?

a) Un alumno opina que hay que empezar con tres ideas del autor que para él son las más importantes, porque de ahí se puede hablar del resto de 
las ideas; hay que buscar en los libros las partes que se relacionan con esas ideas y organizar la información en un cuadro para tener una guía durante la exposición, y hay que explicarlo dando el propio punto de vista.

b) Otro alumno considera que hay que fijarse en las ideas marcadas en los libros de ese autor y consultar con algunos compañeros si marcaron las mismas ideas, si es así hay que irlas anotando en el cuaderno con palabras propias, para después estudiar de ahí y exponerlo en clase.

c) Un alumno opina que hay que ir escribiendo las distintas características que se recuerden de la lectura de los libros de ese autor y asegurarse que estén bien escritas controlando con los libros. Después hay que tratar de grabarlas en la mente para poder decirlas bien cuando exponga.

- Para la categoría condiciones de aprendizaje a partir de la lectura y el escenario leer para ser evaluado:

Dos días antes de un examen, un grupo de alumnos que venían leyendo y estudiando juntos deciden repasar lo que aprendieron cada uno por separado, pero no todos piensan igual sobre cómo hacer el repaso ¿Con quién estás más de acuerdo?

a) Un alumno dice que hay que ir a estudiar a un lugar cómodo y tranquilo, y repasar de nuevo con la vista todos los apuntes, cuadros y resúmenes. Después, leerlos en voz alta.

b) Otro piensa que hay que fijarse en las anotaciones que se hicieron sobre cómo iba a ser el parcial y qué había que tener en cuenta, y repasar los resúmenes y cuadros en base a eso. También sería importante que un compañero que haya estudiado en otro grupo le haga preguntas diferentes a las que ya se hicieron en su grupo.

c) Otro cree que hay que ir diciendo lo que se recuerde y controlando con los apuntes, cuadros y resúmenes si se olvidó algo importante, y si lo que va diciendo con sus palabras es bastante parecido a lo que dicen los textos.

- Para la categoría procesos de aprendizaje a partir de la lectura y el escenario leer para escribir con otros:

Varios alumnos le comentan a su profesor que al revisar los libros para hacer una monografía hay uno que tiene partes que son difíciles de entender. El profesor les pide que lo lean de nuevo para la clase siguiente, así lo discuten entre todos. Un grupo de compañeros se junta a leerlo, pero no se ponen de acuerdo sobre cuál es el mejor modo de realizar la lectura y aparecen en la discusión distintas opiniones sobre lo que hay que hacer. ¿Con cuál estás más de acuerdo?

a) Ver qué nos quiere decir el autor, prestar atención a las partes más importantes y relacionarlas con cosas ya conocidas, interpretar el significado de las palabras desconocidas y cuando algo no se entiende marcarlo en el libro para consultar al profesor en clase.

b) Ir leyendo por partes, buscar en el diccionario las palabras que no se entienden o marcarlas en el libro para preguntárselas al profesor en la clase. También es necesario subrayar y copiar las partes más importantes.

c) Ponerse de acuerdo en cuál va a ser el objetivo del grupo y a medida que se lee ir viendo si lo que dice el libro ya es conocido, para poder ir relacionándolo con lo que se quiere aprender, deteniéndose ante las dificultades que se presenten, intentando superarlas, y en caso de no poder, preguntar en clase sobre lo que no se entiende.

- Para la categoría resultados de aprendizaje a partir de la lectura y el escenario leer para hacer una presentación oral individual:

Distintos estudiantes de primer año hacen exposiciones diferentes sobre las ideas propuestas por un autor que leyeron en libros y artículos de internet. El resto de los compañeros discute sobre cuál fue el estudiante que mostró haber aprendido mejor esas ideas. ¿Con qué opinión estás más de acuerdo?

a) El estudiante que más aprendió fue el que pudo decir la mayor cantidad de ideas propuestas por el autor, y las dijo bastante parecidas a como estaban en los libros y en los artículos.

b) El estudiante que más aprendió es el que indicó las ideas más importantes con sus propias palabras y fue dando ejemplos.

c) El estudiante que más aprendió es el que tomó las ideas centrales propuestas por el autor, las fue explicando y podía relacionarlas con lo que él pensaba y sabía sobre eso.

\section{a.2.2. Estimación de la validez de contenido}

Para determinar si los ítems del instrumento indagaban las dimensiones y categorías propuestas, la validez de contenido se estimó a través de un sistema de control de expertos, que clasificaron y juzgaron la adecuación de las categorías establecidas para cada dimensión de la variable, en función de los fundamentos teóricos y del objetivo del cuestionario. 
Se escogieron tres expertos en el estudio de las concepciones sobre el aprendizaje entendidas como teorías implícitas, aunque con perfiles diferentes: a) investigadora experimentada en el estudio de las teorías implícitas en un dominio cercano al que se indaga en el presente trabajo, el del aprendizaje de la escritura; b) investigador joven recientemente doctorado en el área; y c) investigadora experimentada en el estudio de las teorías implícitas en un dominio diferente al que se indaga en el presente trabajo.

A los tres expertos se les envió el cuestionario acompañado de una consigna en la que se les solicitaba identificar para cada una de las opciones de los dilemas las concepciones, tal como ellas se definen teóricamente (teoría directa, teoría interpretativa y teoría constructiva).

Como resultado del proceso de validación del instrumento se obtuvo un grado de acuerdo elevado. El total de opciones (respuestas posibles) sobre las que tenían que expedirse los expertos fueron 36 . En el $66,66 \%$ de los casos hubo un acuerdo total por parte de los jueces respecto a la categoría asignada a cada opción; esto es: los tres jueces coincidieron con la categoría asignada por nosotros en 24 de las 36 opciones. En el restante 33,33\% de los casos hubo un acuerdo parcial elevado; esto es: se expidieron en acuerdo dos jueces sobre uno, en coincidencia a la categoría asignada por nosotros en 12 de las 36 opciones.

Lo anterior permitió considerar que a partir de las coincidencias, en términos de contenido, el cuestionario es aceptable. No obstante, en los casos en que el acuerdo no fue total, se procedió a una nueva revisión de las opciones para ajustarlas con mayor detalle a las categorías definidas.

Las indicaciones de los expertos permitieron, por una parte, mejorar las expresiones empleadas en el cuestionario y, por otra, otorgar mayor precisión a algunas de las definiciones conceptuales de las dimensiones y categorías que lo orientan.

\section{a.2.3. Estimación de la confiabilidad}

Para determinar la confiabilidad del instrumento, es decir "el grado en que [...] produce resultados consistentes y coherentes" (Hernández Sampieri, Fernández Collado \& Bapista Lucio, 2006, p. 277), se procedió a la entrega de la prueba piloto del cuestionario de dilemas a un grupo de estudiantes universitarios de primer año.

Se administró el cuestionario piloto a una muestra aleatoria de cincuenta estudiantes, ingresantes a la Facultad de Ciencias Humanas, de la Universidad
Nacional de Río Cuarto (República Argentina). Una vez obtenidas las respuestas de los sujetos se procedió al procesamiento estadístico de los datos. Se obtuvo para el coeficiente de confiabilidad de Cronbach un $\alpha=.68$. De acuerdo a este resultado, se entiende que para este tipo de cuestionario, en función de la cantidad de ítems que lo constituyen (12), el alfa obtenido es aceptable (Macchiarola, 2008).

\section{Conclusiones}

En las secciones precedentes hemos ido mostrando cómo se desarrolló el proceso de construcción del cuestionario de dilemas, que indaga las concepciones -entendidas como teorías implícitas- que mantienen los estudiantes universitarios sobre el aprendizaje a partir de la lectura de textos académicos.

Considerando que las teorías implícitas se reflejan en ámbitos prácticos de actuación, hemos abordado este objeto de estudio a partir de la construcción del cuestionario de dilemas, planteando situaciones problemáticas con diferentes opciones de resolución y atendiendo a la configuración de las mismas en diferentes escenarios educativos, que reflejan situaciones prácticas cotidianas vividas por los propios ingresantes universitarios. Confiábamos en que la consideración de estas cuestiones nos acercaría a la indagación de los principios que configuran las teorías implícitas.

Los resultados obtenidos a raiz del proceso de validación y confiabilidad del cuestionario de dilemas elaborado estarían mostrando que el mismo se constituye en un instrumento válido, en cuanto a su contenido, y confiable para ser administrado a estudiantes universitarios ingresantes, con el fin de indagar cuáles son sus concepciones implícitas sobre el aprendizaje a partir de la lectura de textos académicos.

Con lo expuesto, esperamos que la aplicación extendida de este instrumento permita avanzar en la conceptualización y caracterización de las concepciones de los ingresantes universitarios sobre el aprendizaje mediante la lectura y su vinculación con las prácticas lectoras.

No obstante deben señalarse dos limitaciones: la primera, referida al objeto de indagación; dado el carácter complejo e implícito que asumen las concepciones, el estudio de las mismas debe complementarse con otras herramientas de indagación que permitan registrar y analizar las actuaciones 
y respuestas de los sujetos en situaciones 'reales'. La segunda limitación, referida a la construcción deductiva de las categorías y dimensiones de este instrumento, reclama el mismo recaudo, puesto que, tal como señala Macchiarola (2004), las teorías preexistentes pueden restringir las opciones, ocultando matices y aun otras descripciones posibles. De tal modo que para avanzar en descripciones más exhaustivas, necesariamente debemos contemplar instancias de triangulación metodológica, incorporando modalidades de recolección de datos etnográficas y metodologías de análisis inductivas.

\section{Referencias}

Atkinson, T. \& Claxton, G. (2000). El profesor intuitivo. Barcelona: Octaedro, 2002.

Braslavsky, B. (2005). La comprensión del lenguaje escrito. En B. Braslavsky, Enseñar a entender lo que se lee. La alfabetización en la familia y en la escuela (pp. 48-54). México: Fondo de Cultura Económica.

Dubois, M. E. (1987). El Proceso de Lectura. De la Teoría a la Práctica. Buenos Aires: Aique.

Goodman, K. (1994). La lectura, la escritura y los textos escritos: una perspectiva transaccional y sociopsicolingüística. Textos en contexto (2). Los procesos de lectura y escritura. Buenos Aires: Lectura y Vida.

Macchiarola, V. (2004). Concepciones de directivos de escuelas secundarias sobre el planeamiento institucional. El cuestionario de dilemas como procedimiento para su estudio. III Congreso Nacional y I Internacional de Investigación Educativa. Cipoletti: Editorial de la Universidad Nacional del Comahue.

Macchiarola, V. (2008). Metodología. En: V. Macchiarola, Concepciones sobre el planeamiento institucional (pp. 89-116). Río Cuarto: Editorial Universidad Nacional de Río Cuarto.

Pérez Echeverría, M. P., Mateos, M. \& Scheuer, N. (2006). Enfoques en el estudio de las concepciones sobre el aprendizaje y la enseñanza. En J. I. Pozo et al. (Ed.), Nuevas formas de pensar la enseñanza y el aprendizaje. Las concepciones de profesores y alumnos (pp. 55-89). Barcelona: GRAÒ.

Pérez Echeverría, M. P., J. I. Pozo, A. Pecharromán, J. Cervi \& P. Martínez (2006). Las concepciones de los profesores de educación secundaria sobre el aprendizaje y la enseñanza. En J. I. Pozo et al. (Ed.), Nuevas formas de pensar la enseñanza y el aprendizaje. Las concepciones de profesores y alumnos (pp. 289-302). Barcelona: GRAÒ.

Pozo, J. I. \& Gómez Crespo, M. A. (1998). Aprender y enseñar ciencia. Del conocimiento cotidiano al conocimiento científico. Madrid: Morata.

Pozo, J. I. \& Scheuer, N. (1999). Las concepciones sobre el aprendizaje como teorías implícitas. En: J. I. Pozo \& C. Monereo (Coords.), El aprendizaje estratégico (pp. 87-108). Madrid: Santillana.

Pozo, J. I., Scheuer, N., Mateos, M. \& Pérez Echeverría, M. P. (2006). Las teorías implícitas sobre el aprendizaje y la enseñanza. En J. I. Pozo et al. (Ed.), Nuevas formas de pensar la enseñanza y el aprendizaje. Las concepciones de profesores y alumnos (pp. 95-127). Barcelona: GRAÒ.

Pozo J. I. (2009). Adquirir una concepción compleja del conocimiento: Creencias epistemológicas y concepciones de aprendizaje. En Pozo J. I. \& M. del P. Pérez Echeverría (Coords.), Psicología del aprendizaje universitario: La formación en competencias (pp. 70-80). Madrid: Morata.

Rinaudo, M. C. (1999). La naturaleza del proceso de comprensión de textos. En: M. C. Rinaudo, Comprensión del texto escrito (pp. 13-24). Río Cuarto: Editorial de la Fundación Universidad Nacional de Río Cuarto.

Rodrigo, M. J., Rodríguez, A. \& Marrero, J. (1993). Las teorías implícitas. Una aproximación al conocimiento cotidiano. Madrid: Visor.

Rosenblatt, L. M. (1994). La teoría transaccional de la lectura y la escritura. Textos en contexto $\mathrm{N}^{\circ} \mathrm{I}$. Los procesos de lectura y escritura. Lectura y Vida. Buenos Aires. 1996. 16-71.

Hernández Sampieri, R., Fernández Collado, C. \& Bapista Lucio, P. (2006). Recolección de datos cuantitativos. En: R. Hernández Sampieri, C. Fernández Collado \& P. Bapista Lucio, Metodología de la investigación (pp. 273-404). México: McGraw-Hill Interamericana.

Teberosky, A. (1997). El conocimiento cotidiano, escolar y científico en el dominio del lenguaje escrito. En M. J. Rodrigo \& J. Arnay (Comp.), La construcción del conocimiento escolar (pp. 243-262). Barcelona: Paidós.

Vilanova S. L., García, M. B. \& Señoriño, O. (2007). Concepciones acerca del aprendizaje: diseño y validación de un cuestionario para profesores en formación. Revista Electrónica de Investigación Educativa, 9 (2). Consultado el 15 de marzo de 2009, en http://redie.uabc.mx/vo19no2/contenido-vilanova. html 